\title{
Estudo dos Parâmetros Psicométricos da Escala de Vulnerabilidade ao Estresse no Trabalho
}

\author{
Fabiano Koich Miguel ${ }^{1 *}$ - Ana Paula Porto Noronha* \\ *Universidade São Francisco, Itatiba, Brasil
}

\begin{abstract}
Resumo. A presente pesquisa teve como objetivo estudar os parâmetros psicométricos da Escala de Vulnerabilidade ao Estresse no Trabalho (EVET) por relação com outro instrumento que avalia estresse, o Inventário de Sintomas de Stress para Adultos de Lipp (ISSL). Participaram da pesquisa 116 pessoas, sendo 85 do sexo feminino, com idade variando de 19 a 67 anos. A EVET não apresentou correlação significativa com idade e escolaridade, nem apresentou diferença significativa entre os sexos. A relação entre EVET e ISSL revelou correlações significativas e positivas entre os instrumentos. Mais especificamente, a EVET mostrou-se sensível para diferenciar sujeitos que se encontravam na fase de exaustão do estresse daqueles sem estresse. Quanto à precisão da EVET, o alfa de Cronbach foi de 0,90. Palavras-chave: estresse laboral; parâmetros psicométricos; testes psicológicos; construção de instrumento.
\end{abstract}

\begin{abstract}
The present research studied psychometric parameters for the Escala de Vulnerabilidade ao Estresse no Trabalho (EVET) by studying its psychometrical parameters and correlating it with another test that assess stress, the Inventário de Sintomas de Stress para Adultos de Lipp (ISSL). The participants were 116 people, being 85 women, with ages raging from 19 to 67 . The EVET showed no significant correlations with age and school level, nor showed significant differences between sexes. Upon correlation with the ISSL, numerous significant positive correlations were found. More specifically, EVET showed sensitivity to separate individual that were in the stress' exhaustion stage from those with no stress. The EVET's reliability, Cronbach's alpha, was 0,90. Keywords: work stress; psychometric parameters; psychological tests; test making.
\end{abstract}

\section{Introdução}

O termo estresse (vocábulo português para stress) tem sido amplamente utilizado por leigos e costuma ser compreendido pela maioria das pessoas na linguagem cotidiana, referindo-se a uma situação que causa pressão e desconforto sobre o indivíduo (Haan, 1982).

\footnotetext{
${ }^{1}$ Por favor dirigir la correspondencia relacionada con este artículo a: Fabiano Koich Miguel

Universidade São Francisco, Itatiba, Brasil

E-mail: fabianokm@yahoo.com
} 
A definição do conceito, proposta pelo fisiologista Selye (1982) na década de 1930, foi de um resultado não-específico de qualquer demanda sobre o organismo, podendo o efeito ser tanto mental quanto somático. As demandas que eliciam as respostas de estresse foram chamadas de agentes estressores. O estresse apareceria, portanto, como resultado de uma transação difícil entre a pessoa e o ambiente (Lease, 1999).

No que se refere à definição proposta por Selye (1982), entende-se por resultado nãoespecífico a impossibilidade de se apontar um fator único como a causa da reação de estresse. O autor relacionou uma série de situações distintas que podem gerar estresse, a saber, excitação emocional, esforço excessivo, fadiga, dor, medo, concentração, humilhação ou até mesmo sucesso grande e inesperado. $\mathrm{O}$ autor considerou que excitação psicológica poderia ser considerada como um dos mais freqüentes eliciadores do quadro. Complementando, Perkins (1982) chama a atenção para o fato de que não são apenas eventos catastróficos que provocam desordem e estresse, mas os eventos cotidianos também podem acarretar algum estresse e risco, de maneira que a sucessão de eventos estressores, mesmo que estes sejam brandos, pode se tornar cumulativa e provocar efeitos na resistência do organismo. Nesse sentido, o estresse, assim como grande parte das doenças, não apareceria de uma hora para outra.

Embora existam diferenças individuais no que diz respeito à maneira como as pessoas enfrentam seus problemas, o organismo tem uma tendência a responder às solicitações de maneira estereotipada. Dessa forma, para as pessoas enfrentarem qualquer tipo de demanda crescente da atividade vital, há mudanças bioquímicas semelhantes (Selye, 1982). Entre os sintomas que aparecem no quadro de estresse, destacam-se as seguintes manifestações físicas: maior circulação de sangue, com coração batendo acelerado e respiração mais rápida; palidez, com mãos e pés frios, devido ao sangue ter sido retirado das zonas periféricas; aumento da sudorese; tensão muscular, que pode levar a contrações musculares involuntárias; diminuição da resistência imunológica; e redução de outras atividades não ligadas à sobrevivência imediata, como a digestão (Delboni, 1997; Lipp, 1996; Lipp \& cols., 1987).

Ainda, o desgaste provocado pelo estado de alerta pode levar à redução da libido e dificuldade em se concentrar numa tarefa. O sujeito não consegue encadear suas idéias de maneira adequada, tendo dificuldades em desenvolver uma linha de raciocínio lógica (Lipp \& Tanganelli, 2002). O cansaço mental também aparece por meio de distúrbios do sono, podendo ser tanto insônia como sono excessivo, e freqüentemente o sono é agitado (Delboni, 
1997). Começam a aparecer desânimo e desmotivação, uma vez que o sujeito não se vê capaz de resolver seus problemas. A auto-estima diminui, frente à sensação de impotência que esse estado transmite, e o sujeito passa a se considerar subqualificado. As sensações de fragilidade, solidão e desamparo podem vir a favorecer o surgimento da depressão (Lipp e cols., 1987).

Ao longo do desenvolvimento do estresse, surgem três fases ou estágios, a saber, alerta, resistência e exaustão, sendo que cada um deles possui suas próprias características psicológicas e fisiológicas. A época do surgimento e a duração de cada fase dependem da intensidade do evento estressor, do seu tempo de permanência e do seu efeito cumulativo, além da maneira como o indivíduo lida com a situação (Zegans, 1982).

A fase de alerta inicia-se com a reação de alerta ou alarme do sujeito frente ao evento considerado estressor. Selye (1982) havia chamado de reação de alarme à resposta inicial do quadro de estresse, em que ocorre a ruptura do equilíbrio interno do organismo (homeostase), com preparo para luta ou fuga. O sujeito fica em estado de prontidão para solucionar alguma emergência. Se o perigo for eliminado, o organismo retorna à sua condição normal de relaxamento, a adrenalina é reduzida e a homeostase é restaurada, de maneira que os sintomas desaparecem.

Se a exposição ao agente estressor que propicia a reação de alarme permanece, ou se o indivíduo não dispõe de habilidade para lidar com ele, após algum tempo o organismo entra na fase de resistência (Selye, 1982). Nessa fase, o corpo procura restabelecer sua homeostase por meio de adaptação ao estado de estresse e consome as reservas de energia adaptativa. Os sintomas que aparecem são bastante diferentes, em muitos aspectos opostos, aos que se manifestaram na primeira fase, os quais costumam desaparecer. Sintomas característicos da fase de resistência são problemas com memória, sensação constante de cansaço, excesso de irritabilidade e sentimentos de dúvida quanto às próprias capacidades, entre outros (Lipp, 2000).

Gastando-se toda a energia adaptativa, devido ao fato do sujeito não ter capacidade para lidar com a tensão, ou também com a ocorrência concomitante de outros eventos estressores nocivos, entra-se na fase de exaustão, na qual ocorre aumento das estruturas linfáticas e exaustão tanto física quanto psicológica, sendo esta última normalmente na forma de depressão (Selye, 1982). É nessa fase que aparecem doenças crônicas, como infarto do miocárdio, hipertensão arterial crônica ou mesmo doenças mentais, além de sintomas 
psicossomáticos, como úlceras e colite, resultados do elevado caráter patogênico das mudanças fisiológicas e bioquímicas (Reinhold, 1996).

Normalmente, durante o curso da vida, as pessoas passam pelas duas primeiras fases do estresse diversas vezes, e é dessa maneira que o indivíduo vai se tornando adaptado às atividades e demandas típicas da espécie humana. Porém, apesar de sono e descanso serem capazes de restaurar a resistência e adaptabilidade do organismo após atividades de intenso estresse, essa restauração nunca chega a ser completa. Dessa forma, o corpo carrega algumas marcas químicas irreversíveis, cujo acúmulo compõe o sinal de envelhecimento (Selye, 1982).

No que diz respeito à tolerância ao estresse, as pessoas podem apresentar níveis diferentes, sendo que algumas são mais resistentes do que as outras. De acordo com Delboni (1997), quanto melhor um indivíduo reagir aos eventos estressores, menos sintomas desenvolverá. Aqueles que possuem maior resistência ao estresse, segundo Lipp e colaboradores (1987), apresentam algumas características em comum, como abertura e aceitação de situações novas, maneira positiva de encarar os acontecimentos da vida, envolvimento com trabalho, entre outras.

Em relação ao estresse no ambiente de trabalho, também chamado de estresse laboral ou ocupacional, os estudos são relativamente recentes, embora já seja conhecida há muito tempo a relação entre perda da qualidade de vida e condições prejudiciais como pressão, relacionamento difícil com chefia, situações de risco, entre outros (Holt, 1982). O surgimento do campo de estudo se deu principalmente graças ao movimento de preocupação com a saúde mental, passando-se a considerar que existem aspectos deletérios no local de trabalho que podem acarretar efeitos psicológicos. O estresse laboral pode ser definido como demandas relacionadas ao ambiente de trabalho, cujo enfrentamento inadequado, que depende das características individuais, pode levar aos resultados negativos do estresse, ou seja, mal-estar físico e/ou psicológico (Clarke \& Cooper, 2000; Edwards \& Burnard, 2003).

No que se refere ao trabalho, Delboni (1997) considera que o responsável por uma organização também é responsável pelo bem-estar daqueles que trabalham ali. É função dos dirigentes da empresa fomentar programas e benefícios que visem reduzir os fatores que podem provocar estresse. Dessa maneira, a produtividade da empresa aumenta, uma vez que seus funcionários se encontram mais satisfeitos e com melhores condições de vida. Segundo a autora, o estresse ocupa papel de destaque nos programas preventivos nas empresas em todo 
mundo. Isso porque se considera que ele representa um custo alto para as organizações, já que as horas de trabalho perdidas, absenteísmo e gastos com assistência médica acarretam queda da produtividade.

Nesse contexto, muitas pesquisas têm buscado elencar os eventos estressores no ambiente de trabalho. Num estudo com 12 professores de inglês como segunda língua, Markham (2000) encontrou que todos eles apresentavam estresse devido à característica desafiadora do trabalho, embora também relatassem satisfação com o emprego. Entre os estressores estavam trabalhar com alunos atrasados na série escolar e lecionar numa classe com alunos em diferentes níveis de proficiência em inglês.

Barnes, Agago e Coombs (1998) verificaram os níveis de satisfação de docentes, considerando como possíveis estressores a remuneração, reputação na instituição, compromisso com o horário, influência institucional e interação com estudantes, e avaliaram sua relação com intenção de deixar o local de trabalho. Encontrou-se que essa relação era mediada pelo interesse na própria disciplina e pelo senso de comunidade, consideradas variáveis de ajustamento.

Em uma pesquisa avaliando a qualidade de vida de juízes, Lipp e Tanganelli (2002) encontraram que os principais estressores foram sobrecarga de trabalho e interferência com a vida familiar. Em relação às estratégias de enfrentamento do estresse, a mais mencionada pelos participantes foi conversar com o cônjuge ou outra pessoa afetivamente próxima.

Analisando os níveis de estresse de docentes, Lease (1999) buscou verificar a afirmação comum de que mulheres com menos tempo de experiência no trabalho estariam mais sujeitas ao estresse ocupacional. $O$ autor encontrou que não houve diferenças significativas, tanto para o sexo quanto para o tempo de trabalho.

Bartell (1996) estudou o nível de estresse de psicólogos escolares, considerando a escola como um ambiente potencialmente estressor. Numa escala de 1 a 5 , sendo 1 considerado baixo estresse e 5 , alto, a amostra obteve uma pontuação média de 3,80 , sendo que os estressores mais relatados foram falta de tempo para executar o trabalho adequadamente e superiores incompetentes ou inflexíveis.

$\mathrm{Na}$ área médica, considerada uma das áreas cujos profissionais experimentam maior nível de estresse (Edwards, Burnard, Coyle, Fothergill \& Hanningan, 2001), existem muitas pesquisas com enfermeiros. Os principais eventos considerados estressores para os profissionais de enfermagem são elevada carga de trabalho e contato próximo com a morte ou 
agonia dos pacientes (Tyler, Carroll \& Cunningham, 1991), conflito com médicos e outros enfermeiros (Lees \& Ellis, 1990) e excesso de trabalho, interrupções e carga horária excessiva (Rout, 2000). Nesse sentido, a pesquisa de Healy e McKay (2000) encontrou uma correlação positiva entre estresse e transtornos do humor e correlação negativa entre estresse e satisfação no trabalho.

As diferenças nos níveis de estresse entre sexos têm sido pesquisadas por diversos estudos e seus resultados são diversos, com alguns encontrando maior estresse em homens, outros em mulheres e outros, ainda, não encontrando diferenças significativas (Lease, 1999). Nesse sentido, a pesquisa de Rout (1999) verificou se havia diferenças de sexos entre médicos clínicos gerais. Embora não tenha encontrado diferenças significativas entre os dois grupos, os resultados apontaram para um nível elevado de estresse para ambos, especialmente relacionado à insatisfação com salário, horas de trabalho e quantidade de trabalho.

Estudando motoristas de ônibus públicos, Rydstedt, Johansson e Evans (1998) não encontraram diferenças significativas entres os sexos. Essa profissão, segundo os autores, é considerada como uma das mais suscetíveis ao estresse ocupacional, especialmente devido ao congestionamento de tráfego e, em certos casos, condições perigosas de direção. Também Calais, Andrade e Lipp (2003) pesquisaram a diferença de sexos entre adultos jovens em diversos níveis educacionais. Em todos os grupos, as mulheres apresentaram maiores escores de estresse, e a sintomatologia foi predominantemente psicológica.

No que diz respeito à mensuração do construto, os esforços em pesquisa normalmente se concentram em demonstrar que as conseqüências da má adaptação ao estressor podem ser preditas por meio de escalas de eventos de vida (Holroyd \& Lazarus, 1982). Embora o surgimento do estresse seja dependente do significado que o sujeito atribui para a situação, o que inviabilizaria a construção de uma lista com estímulos estressores específicos, Haan (1982) considera que tais significados possuem certas características que são comuns o suficiente para que se possa construir uma taxonomia de situações estressoras. Nesse sentido, uma lista de eventos de vida poderia fornecer um índice simples do estresse da pessoa. Até mesmo a ausência de um evento, como uma promoção negada ou a recusa num desejável aumento salarial, poderia ser considerada em si como outro evento estressor (Perkins, 1982).

O uso de inventários de auto-relato nas avaliações tende a se mostrar eficiente em termos de custo e tempo, e parte do princípio de que, embora o indivíduo possa estar sujeito a distorções em suas representações, é ele que está mais próximo de descrever sua relação com 
o estressor do que qualquer observador externo (Derogatis, 1982). Com o desenvolvimento das pesquisas, o uso de escalas de eventos de vida, segundo Perkins (1982), acabará sendo substituído por modelos multifatoriais que levam em conta diferenças individuais e o contexto do trabalho.

Dada a escassez de instrumentos de avaliação do estresse no mercado brasileiro, a Escala de Vulnerabilidade ao Estresse no Trabalho (EVET) está sendo estudada por Sisto, Baptista, Noronha e Santos (em construção). O teste se baseia na apresentação de diversas situações relacionadas ao trabalho consideradas geradoras de estresse. O sujeito é solicitado a avaliar o quanto se sente incomodado com cada uma daquelas situações, se elas ocorrem com ele, e pontuar de acordo.

Outro instrumento desenvolvido a fim de se diagnosticar o estresse é o Inventário de Sintomas de Stress para Adultos de Lipp (ISSL), construído por Lipp (2000), sendo que já se encontra aprovado pelo Conselho Federal de Psicologia (CFP, 2006). Trata-se de um questionário que se baseia na sintomatologia do estresse, apresentando quadros com sintomas tanto de estresse físico quanto psicológico, que o sujeito assinala se ocorreram durante determinados períodos de tempo.

Para que os novos instrumentos elaborados e os já existentes no Brasil disponham de aceitação tanto dos psicólogos quanto da comunidade leiga, os testes devem dispor de estudos quanto a sua validade e a sua precisão, as amostras devem ser brasileiras e as instruções de aplicação devem ser claras e objetivas, tanto para o aplicador quanto para o aplicando. $\mathrm{O}$ questionamento a respeito da área e dos testes é salutar e necessário, uma vez que gera pesquisas e aprimoramento dos instrumentos de avaliação psicológica (Noronha, 2002). Levando-se em consideração o referencial teórico apresentado e buscando ampliar os estudos na área, essa pesquisa possuiu o objetivo de estudar os parâmetros psicométricos da EVET por meio de correlação com a ISSL. Como o primeiro teste mede a vulnerabilidade do indivíduo frente a situações estressoras e o segundo faz um inventário dos sintomas apresentados, esperou-se correlação moderada, por se tratarem de construtos relacionados.

\section{Método}

\subsection{Participantes}

Participaram da pesquisa 116 pessoas de locais de trabalho distintos situados em três municípios do estado de São Paulo, incluindo a capital, sendo 85 (72,6\%) do sexo feminino. 
A faixa etária compreendeu dos 19 aos 67 anos, sendo a média 39,60 e desvio-padrão 11,17.

\subsection{Instrumentos}

\subsubsection{Escala de Vulnerabilidade ao Estresse no Trabalho (EVET)}

A EVET, de autoria de Sisto e colaboradores (em construção), tem por finalidade avaliar a vulnerabilidade dos sujeitos quanto à presença de elementos estressores no ambiente de trabalho. Para isso, apresenta itens descrevendo situações consideradas geradoras de estresse, solicitando aos sujeitos que pontuem as situações que causam incômodo seguindo uma escala tipo likert, com opções de nunca (um), às vezes (dois) e freqüentemente (três). Exemplos das situações são: acúmulo de funções, ambiente físico inadequado, expectativa excessiva da chefia, falta de perspectiva profissional, fazer o trabalho do outro, não ser valorizado, salários atrasados, entre outras.

Um estudo preliminar da escala foi publicado por Barbosa, Bighetti, Baptista e Noronha (2005), utilizando 381 estudantes universitários como sujeitos e buscando-se diferenciar os cursos com base nas respostas. Dos 154 itens originalmente propostos para o instrumento, 73 permitiram a diferenciação dos cursos. Notou-se também que os sujeitos da área de saúde são mais suscetíveis aos eventos estressores quando comparados com outras áreas, havendo predominância da área de enfermagem, que se diferenciou das demais em 63 itens.

Um novo estudo feito pelos autores a partir da escala com 73 itens verificou a estrutura interna do instrumento, reduzindo o número de itens para 40. Foram também identificados três fatores, denominados Aborrecimento e contrariedade, Pressão no trabalho e Falta de suporte organizacional.

A pontuação da EVET se dá pela soma dos itens assinalados pelos sujeitos. A pontuação mínima é 40 e a máxima, 120. Em relação a cada fator, Aborrecimento e contrariedade possui uma pontuação mínima de 17 e máxima de 51, Pressão no trabalho possui mínima de 13 e máxima de 39 e Falta de suporte organizacional possui mínima de 10 e máxima de 30.

\subsubsection{Inventário de Sintomas de Stress para Adultos de Lipp (ISSL)}

O ISSL é um instrumento que tem como finalidade identificar a sintomatologia apresentada pelo sujeito, ou seja, ele avalia se a pessoa demonstra sintomas de estresse, o tipo 
desse sintoma (físico ou psicológico) e a fase em que ele se encontra, sendo definidas quatro fases de manifestação (Lipp, 2000). A primeira fase é a fase de alerta, em que o indivíduo se prepara para uma reação de enfrentamento ou fuga; a segunda, chamada resistência, aparece se há uma permanência da primeira fase por tempo indeterminado, iniciando-se uma adaptação do organismo aos eventos estressores, sendo portanto acompanhada de sensação de desgaste e fadiga; a terceira e quarta fases surgem quando o organismo esgota sua reserva de energia adaptativa, aparecendo, então, a exaustão e doenças, sendo que na terceira as doenças ainda não são tão graves e a pessoa ainda consegue trabalhar e se relacionar socialmente.

Segundo Lipp (2000), a ISSL foi validada por meio de análise de consistência interna e análise fatorial, numa amostra de 1843 pessoas. Encontrou-se alfa de Cronbach de 0,91 para a escala geral, e a análise fatorial separou dois eixos, sendo que o primeiro incluía itens de natureza psicológica e o segundo, de natureza física. Realizando análise qualitativa, percebeu-se que havia uma diferença substancial entre as pessoas na fase de resistência que possuíam alguns sintomas e aquelas com elevado número de sintomas. Por meio de análise de agrupamento dos itens, duas classes para essa fase foram encontradas, o que levou à divisão em fase de resistência e quase-exaustão.

O manual do ISSL (Lipp, 2000) fornece o procedimento para se calcular as porcentagens de sintomas de cada quadro e também de sintomas físicos e psicológicos. Por exemplo, no caso do quadro 1, se o sujeito assinalou até 6 sintomas, a porcentagem atribuída é zero. De sete sintomas para cima, utiliza-se uma tabela do manual para converter o número de sintomas para porcentagem. Na presente pesquisa, contudo, optou-se por não utilizar esse método de correção, e sim fazer uso dos resultados brutos, ou seja, o número de sintomas assinalados em cada quadro e não sua porcentagem.

\section{Resultados}

\subsection{Análise descritiva}

A pontuação média na EVET foi 70,66, com desvio-padrão de 12,96. Os dados referentes aos três fatores da escala também são apresentados na Tabela 1. 


\section{Tabela 1}

Estatística descritiva da EVET

\begin{tabular}{lcccccc}
\hline & \multicolumn{3}{c}{ Medida de Tendência Central } & \multicolumn{3}{c}{ Medida de Dispersem } \\
\cline { 2 - 7 } & Média & Mediana & Moda & $\begin{array}{c}\text { Desvio- } \\
\text { padrão }\end{array}$ & Mínimo & Máximo \\
\hline $\begin{array}{l}\text { EVET1. } \\
\text { Aborrecimento e contrariedade }\end{array}$ & 31,31 & 30,50 & 27 & 7,30 & 17 & 50 \\
$\begin{array}{l}\text { EVET2. } \\
\text { Pressão no trabalho }\end{array}$ & 24,11 & 24,00 & 25 & 5,83 & 12 & 38 \\
$\begin{array}{l}\text { EVET3. } \\
\text { Falta de suporte organizacional }\end{array}$ & 15,24 & 14,50 & 14 & 3,29 & 10 & 25 \\
Total EVET & 70,66 & 71,00 & 71 & 12,96 & 40 & 107 \\
\hline $\mathrm{N}=116$ & & & & & &
\end{tabular}

A pontuação total mínima na EVET foi de 40 pontos, que coincide com o escore mínimo possível. A pontuação máxima, entretanto, foi de 107, ou seja, nenhum sujeito obteve o escore máximo possível para a EVET, que é de 120. Em relação aos fatores, ocorreu pontuação mínima para os três. No caso do Aborrecimento e contrariedade da Pressão no trabalho, a pontuação máxima ficou apenas um ponto abaixo do escore máximo possível, sugerindo que há participantes que se sentem extremamente incomodados com as situações relacionadas aos respectivos fatores.

A Tabela 2 apresenta a descrição estatística do ISSL. As fases do estresse foram numeradas de 0 a 4, atribuindo-se 0 para ausência de estresse, 1 para fase de alerta, 2 para fase de resistência, 3 para quase-exaustão e 4 para fase de exaustão.

De acordo com o ISSL, 55 pessoas $(47,4 \%)$ foram consideradas como não portadoras de estresse, nenhuma se apresentou na fase de alerta, 56 pessoas (48,3\%) apresentaram-se na fase de resistência, quatro $(3,4 \%)$ na fase de quase-exaustão e apenas uma $(0,9 \%)$ na fase de exaustão. O quadro 1 possui 15 sintomas e a média assinalada pelos sujeitos foi de 2,69 $(\mathrm{DP}=2,15)$. Esse valor tão reduzido explica a ausência de sujeitos na primeira fase do estresse, uma vez que os outros quadros, que representam as outras fases, foram mais assinalados. 


\section{Tabela 2}

Estatística descritiva dos números de sintomas do ISSL

\begin{tabular}{lcccccc}
\hline & \multicolumn{3}{c}{ Medida de Tendência Central } & \multicolumn{3}{c}{ Medida de Dispersem } \\
\cline { 2 - 7 } & Média & Mediana & Moda & $\begin{array}{c}\text { Desvio- } \\
\text { padrão }\end{array}$ & Mínimo & Máximo \\
\hline Fase do estresse (ISSL) & 1,10 & 2,00 & 2 & 1,08 & 0 & 4 \\
Total quadro 1 & 2,69 & 2,00 & 2 & 2,15 & 0 & 9 \\
Total quadro 2 & 3,67 & 4,00 & 4 & 2,77 & 0 & 11 \\
Total quadro 3 & 3,69 & 3,00 & 0 & 3,15 & 0 & 14 \\
Sintomas físicos & 5,43 & 5,00 & 3 & 4,09 & 0 & 19 \\
Sintomas psicológicos & 4,62 & 4,00 & 0 & 3,72 & 0 & 16 \\
Total ISSL & 10,05 & 9,50 & 12 & 7,14 & 0 & 32 \\
\hline
\end{tabular}

$\mathrm{N}=116$

\subsection{Correlações entre EVET e critérios}

A fim de verificar se havia alguma relação entre os escores da EVET e idade, foi realizada correlação. Não foram encontradas correlações significativas entre idade e a EVET, tanto para o escore geral quanto para os três fatores. Na literatura levantada para a presente pesquisa também não foram encontradas referências a diferenças de níveis de estresse de acordo com a idade. Esses dados sugerem, portanto, que a vulnerabilidade ao estresse pode estar presente no sujeito independentemente da idade.

Também foi realizada correlação entre os escores da EVET e escolaridade. Assim como no caso da idade, não houve correlação significativa entre estresse laboral segundo a EVET e escolaridade, com exceção do segundo fator, Pressão no trabalho, que apresentou correlação $r=-0,18(p<0,05)$. Trata-se de um índice muito baixo de correlação, mas que ainda assim sugere que pessoas de maior escolaridade tendem a pontuar menos nesse fator. Não obstante, de maneira geral não se pode dizer que níveis diferentes de escolaridade apresentam níveis variados de estresse.

A fim de se verificar se havia diferenças de médias significativas entre os sexos em relação ao total da EVET e seus fatores, foi realizado teste-t de Student. Encontrou-se diferença significativa de média entre sexos apenas para o segundo fator da EVET, Pressão no trabalho. Os homens obtiveram média de 22,26, enquanto as mulheres de 24,79, sugerindo que elas tendem a se sentir mais incomodadas do que os homens em relação à pressão no 
trabalho. Isso está de acordo com outras pesquisas que verificaram que as mulheres tendem a desenvolver maior estresse no ambiente de trabalho, embora ainda não exista um consenso quanto a qual sexo é mais suscetível aos eventos estressores (Calais e cols., 2003; Lease, 1999).

\subsection{Correlações entre EVET e ISSL}

A fim de se verificar a relação entre os construtos, a EVET foi correlacionada com o ISSL. Na Tabela 3 estão apresentados os resultados das correlações.

\section{Tabela 3.}

Correlações entre EVET e número de sintomas do ISSL $(\mathrm{N}=116)$

\begin{tabular}{lcccc}
\hline & $\begin{array}{c}\text { EVET1. } \\
\text { Aborrecimento } \\
\text { contrariedade }\end{array}$ & $\begin{array}{c}\text { EVET2. } \\
\text { Pressão no } \\
\text { trabalho }\end{array}$ & $\begin{array}{c}\text { EVET3. } \\
\text { Falta de suporte } \\
\text { organizacional }\end{array}$ & Total EVET \\
\hline Fase do estresse (ISSL) & 0,17 & 0,15 & 0,12 & $0,20^{*}$ \\
Total quadro 1 & 0,17 & $-0,05$ & 0,08 & 0,10 \\
Total quadro 2 & 0,14 & 0,16 & 0,13 & 0,18 \\
Total quadro 3 & $0,32^{* * *}$ & 0,16 & 0,16 & $0,29^{* * *}$ \\
Sintomas físicos & $0,22^{*}$ & 0,09 & $0,21^{*}$ & $0,22^{*}$ \\
Sintomas psicológicos & $0,23^{*}$ & 0,13 & 0,04 & $0,20^{*}$ \\
Total ISSL & $0,25^{* *}$ & 0,12 & 0,14 & $0,23^{*}$ \\
\hline * Correlação significativa no nível $\mathrm{p}<0,05$ & & & \\
$* *$ Correlação significativa no nível $\mathrm{p}<0,01$ & & & \\
$* * *$ Correlação significativa no nível $\mathrm{p}<0,001$ & &
\end{tabular}

Os escores dos três fatores da EVET não apresentaram correlação significativa com a fase do estresse segundo o ISSL. Contudo, o escore total da EVET apresentou correlação significativa, embora baixa, sendo $r=0,20(p<0,05)$. Isso indica uma tendência a quanto maior o escore na EVET, mais avançada a fase do estresse. A correlação baixa, porém, sugere que os instrumentos estão avaliando construtos não totalmente semelhantes, mas relacionados. Tal fato foi previsto na hipótese inicial, uma vez que a EVET busca analisar o estresse laboral (Sisto e cols., em construção), enquanto que o ISSL mede sintomas associados ao quadro de estresse (Lipp, 2000).

O escore total da EVET apresentou correlação significativa com o número total de sintomas assinalados no ISSL $(\mathrm{r}=0,23 ; \mathrm{p}<0,05)$. Isso significa que, conforme as pessoas apresentam escores mais altos na EVET, tendem a assinalar mais sintomas de estresse no ISSL. O total da EVET também se correlacionou com a quantidade de sintomas do quadro 3 
$(\mathrm{r}=0,29 ; \mathrm{p}<0,001)$, sugerindo que, quanto mais alta é a pontuação do sujeito na EVET, mais ele assinala sintomas da fase de exaustão. Outras correlações encontradas para o escore total da EVET foram com quantidade de sintomas físicos $(r=0,22 ; \mathrm{p}<0,05)$ e quantidade de sintomas psicológicos $(\mathrm{r}=0,20 ; \mathrm{p}<0,05)$.

O primeiro fator da EVET, Aborrecimento e contrariedade, obteve correlações significativas com as mesmas características do ISSL que o escore geral, porém os índices dessas correlações tenderam a ser um pouco maiores. O segundo fator da EVET, Pressão no trabalho, não apresentou correlações significativas com nenhuma característica do ISSL. O terceiro fator, Falta de suporte, apresentou correlação significativa apenas com a quantidade de sintomas físicos, sendo $\mathrm{r}=0,21(\mathrm{p}<0,05)$.

Como foi encontrado que o escore total da EVET se correlacionou com a quantidade de sintomas relacionados à exaustão, sugeriu-se que a EVET talvez seja sensível para identificar sujeitos na última fase do estresse. Foi realizado também teste-t de Student a fim de se verificar se havia diferenças de médias para dois grupos extremos, a saber, os sujeitos sem estresse e os sujeitos na fase de quase-exaustão ou exaustão. O resultado da prova estatística encontra-se na Tabela 4.

\section{Tabela 4}

Teste-t da EVET em relação à fase do estresse

\begin{tabular}{|c|c|c|c|c|c|}
\hline & \multicolumn{2}{|c|}{ Grupo } & \multirow[b]{3}{*}{$t$} & \multirow[b]{3}{*}{$g l$} & \multirow[b]{3}{*}{$p$} \\
\hline & $\begin{array}{l}\text { Sujeitos sem } \\
\text { estresse }\end{array}$ & $\begin{array}{l}\text { Sujeitos nas } \\
\text { últimas fases }\end{array}$ & & & \\
\hline & Médias & Médias & & & \\
\hline $\begin{array}{l}\text { EVET1. } \\
\text { Aborrecimento e contrariedade }\end{array}$ & 30,20 & 36,40 & $-1,90$ & 58 & 0,063 \\
\hline $\begin{array}{l}\text { EVET2. } \\
\text { Pressão no trabalho }\end{array}$ & 23,20 & 25,60 & $-0,92$ & 58 & 0,360 \\
\hline $\begin{array}{l}\text { EVET3. } \\
\text { Falta de suporte organizacional }\end{array}$ & 14,93 & 18,20 & $-2,20$ & 58 & 0,032 \\
\hline Total EVET & 68,33 & 80,20 & $-2,05$ & 58 & 0,045 \\
\hline
\end{tabular}

Corroborando os dados encontrados na correlação entre EVET e ISSL, o teste-t mostrou que os indivíduos que se encontram na fase de quase-exaustão ou exaustão possuem pontuação muito maior na EVET $(\mathrm{M}=80,20$; $\mathrm{DP}=10,35)$ do que os sujeitos que não possuem estresse $(M=68,33 ; D P=12,56)$. Encontrou-se também diferença de médias significativa para 
os escores no fator 3, Falta de suporte organizacional. Os sujeitos sem estresse obtiveram média 14,93 (DP=3,17) e os sujeitos na fase de quase-exaustão ou exaustão obtiveram média $18,20(\mathrm{DP}=3,42)$. Esse dado demonstra que a escala se mostrou sensível em diferenciar sujeitos não-estressados daqueles com alto nível de estresse.

\subsection{Precisão da EVET}

Foi realizado estudo de precisão da EVET, tanto para a escala como para os itens referentes aos seus três fatores. $O$ índice de precisão alfa de Cronbach para a escala inteira foi de 0,90, o que pode ser considerado ótimo (Conselho Federal de Psicologia, 2003; Cronbach, 1996). De acordo com os resultados, nenhum item aumentaria a precisão da escala se fosse excluído. As correlações item-total variaram de 0,15 a 0,59.

No que diz respeito aos fatores isoladamente, o alfa do fator Aborrecimento e contrariedade foi de 0,87, do fator Pressão no trabalho foi de 0,87 e do fator Falta de suporte organizacional foi de 0,68. Este último mostrou um índice abaixo do recomendado 0,70 (Prieto \& Muniz, 2000), o que talvez possa ser explicado pela reduzida quantidade de itens. Dessa forma, interpretações baseadas apenas neste fator devem ser feitas com cautela.

\section{Discussão}

O objetivo da presente pesquisa foi estudar os parâmetros psicométricos da EVET, relacionando-a a outro instrumento que mensura estresse, qual seja, o ISSL. Foram encontradas correlações significativas entre o escore geral da EVET e os fatores 1 e 3 com a fase do estresse, com o total de sintomas do quadro de quase-exaustão e exaustão, com os sintomas físicos e psicológicos e com o total de sintomas assinalados no ISSL. Em contrapartida, o segundo fator da EVET, Pressão no trabalho, não obteve correlação significativa com nenhum dado do ISSL. Não obstante, as correlações significativas dos

outros fatores e do escore total com o ISSL foram numerosas e todas positivas. Tais correlações foram de baixas a moderadas $(\mathrm{r}=0,20$ a $\mathrm{r}=0,32)$, tendo isso sido previsto na hipótese inicial, uma vez que a EVET busca mensurar a vulnerabilidade do sujeito aos eventos estressores no local de trabalho, enquanto que o ISSL faz um inventário dos sintomas relacionados às fases do estresse. Contudo, a fim de se verificar a validade de construtos relacionados, novas pesquisas são sugeridas com outros instrumentos que avaliem estresse, verificando-se se índices de correlação maiores são encontrados. Nesta pesquisa, os resultados de provas estatísticas entre os sujeitos sem estresse e aqueles com sintomas das 
fases de quase-exaustão ou exaustão apontaram para uma sensibilidade da EVET no que diz respeito a diferenciar esses dois grupos.

Em relação ao sexo, a EVET de maneira geral não demonstrou diferenciação entre homens e mulheres. Não existe um consenso na literatura quanto à maior ou menor vulnerabilidade de um sexo em relação ao estresse, sendo às vezes encontrado que homens desenvolvem mais estresse, outras vezes mulheres e outras vezes nenhuma diferença é encontrada (Calais e cols., 2003; Lease, 1999; Rout, 1999; Rydstedt e cols., 1998). Portanto, talvez a não-diferenciação entre os sexos possa ser uma vantagem, permitindo que a EVET seja aplicada em diversos tipos de ambiente de trabalho.

No que diz respeito à precisão da EVET, a escala como um todo obteve um alfa de Cronbach de 0,90. Tal índice é considerado ótimo pela literatura (CFP, 2003; Cronbach, 1996).

Como afirma Noronha (2002), é pelo constante questionamento da área que novos instrumentos de avaliação podem ser desenvolvidos e a compreensão teórica sobre os fenômenos pode ser ampliada. Nesse sentido, espera-se que essa pesquisa tenha contribuído para o contexto atual da avaliação psicológica, no sentido de estudar, por meio da pesquisa científica, os parâmetros psicométricos de um instrumento de avaliação psicológica em desenvolvimento.

\section{Referências}

Barbosa, M. A., Bighetti, C. A., Baptista, M. N., \& Noronha, A. P. P. (2005). Eventos Estressores Relacionados ao Trabalho: Um Estudo com Universitários. Anais do Encontro Mineiro de Avaliação Psicológica, Minas Gerais, 42-43.

Barnes, L. L. B., Agago, M. O., \& Coombs, W. T. (1998). Effects of Job-related Stress on Faculty Intention to Leave Academia. Research in Higher Education, 39(4), 457-469.

Bartell, R. (1996). Stressors of School Psychologists: A Canadian Perspective. Paper presented at the XIX International School Psychology Colloquium, Eger, Hungria.

Calais, S. L., Andrade, L. M. B., \& Lipp, M. E. N. (2003). Diferenças de Sexo e Escolaridade na Manifestação de Stress em Adultos Jovens. Psicologia: Reflexão e Crítica, 16(2), 257-263.

Clarke, S. G., \& Cooper, C. L. (2000). The Risk Management of Occupational Stress. Health, Risky \& Society, 2(2), 173-187. 
Conselho Federal de Psicologia - CFP (2003). Resolução nº 002/2003 [On-line]. Disponível: http://www.pol.org.br.

Conselho Federal de Psicologia - CFP (2006). Lista de Testes com Parecer Favorável [Online]. Disponível: http://www.pol.org.br/satepsi/sistema/admin.cfm.

Cronbach, L. J. (1996). Fundamentos da Testagem Psicológica. Porto Alegre: Artmed.

Delboni, T. H. (1997). Vencendo o Stress: Como Melhorar as Relações de Trabalho para Viver Melhor. São Paulo: Makron Books.

Derogatis, L. R. (1982). Self-report Measures of Stress. Em L. Goldberger \& S. Breznitz (Eds.), Handbook of Stress: Theoretical and Clinical Aspects. (pp. 270-294). New York: Free Pass.

Edwards, D., \& Burnard, P. (2003). A Systematic Review of Stress and Stress Management Interventions for Mental Health Nurses. Journal of Advanced Nursing, 42(2), 169200.

Edwards, D., Burnard, P., Coyle, D., Fothergill, A., \& Hannigan, B. (2001). A Stepwise Multivariate Analysis of Factors that Contribute to Stress for Mental Health Nurses Working in the Community. Journal of Advanced Nursing, 36(6), 805-813.

Haan, N. (1982). The Assessment of Coping, Defense and Stress. Em L. Goldberger \& S. Breznitz (Eds.), Handbook of Stress: Theoretical and Clinical Aspects. (pp. 254-269). New York: Free Pass.

Healy, C. M., \& McKay, M. F. (2000). Nursing Stress: The Effects of Coping Strategies and Job Satisfaction in a Sample of Australian Nurses. Journal of Advanced Nursing, $31(3), 681-688$.

Holroyd, K. A., \& Lazarus, R. S. (1982). Stress, Coping, and Somatic Adaptation. Em L. Goldberger \& S. Breznitz (Eds.), Handbook of Stress: Theoretical and Clinical Aspects. (pp. 21-35). New York: Free Pass.

Holt, R. R. (1982). Occupational Stress. Em L. Goldberger \& S. Breznitz (Eds.), Handbook of Stress: Theoretical and Clinical Aspects. (pp. 419-444). New York: Free Pass.

Lease, S. H. (1999). Occupational Role Stressors, Coping, Support, and Hardiness as Predictors of Strain in Academic Faculty: An Emphasis on New and Femanel Faculty. Research in Higher Education, 40(3), 285-307.

Lees, S., \& Ellis, N. (1990). The Design of a Stress-management Programme for Nursing Personnel. Journal of Advanced Nursing, 15, 946-961. 
Lipp, M. E. N. (1996). Stress: Conceitos Básicos. Em M. E. N. Lipp (Org.), Pesquisas sobre Stress no Brasil: Saúde, Ocupações e Grupos de Risco. (pp 17-31). Campinas: Papirus.

Lipp, M. E. N., \& Tanganelli, M. S. (2002). Stress e Qualidade de Vida em Magistrados da Justiça do Trabalho: Diferenças entre Homens e Mulheres. Psicologia: Reflexão e Crítica, 15(3), 537-548.

Lipp, M. N. (2000). Manual do Inventário de Sintomas de Stress para Adultos de Lipp (ISSL). São Paulo: Casa do Psicólogo.

Lipp, M. N., \& cols. (1987). Como Enfrentar o Stress. São Paulo: Ícone.

Markham, P. L. (2000). Stressors and Coping Strategies of ESL Teachers. Jounral of Instructional Psychology, 26(4), 268-279.

Noronha, A. P. P. (2002). Os Problemas Mais Graves e Mais Freqüentes no Uso dos Testes Psicológicos. Psicologia: Reflexão e Crítica, 15(1), 135-142.

Perkins, D. V. (1982). The Assessment of Stress Using Life Event Scales. Em L. Goldberger \& S. Breznitz (Eds.), Handbook of Stress: Theoretical and Clinical Aspects. (pp. 320331). New York: Free Pass.

Prieto, G., \& Muñiz, J. (2000). Um Modelo para Evaluar La Calidad de los Tests Utilizados em España [On-line]. Disponível: http://www.cop.es/tests/modelo.htm (acessado em 04/12/01).

Reinhold, H. H. (1996). Stress Ocupacional do Professor. Em M. E. N. Lipp (Org.), Pesquisas sobre Stress no Brasil: Saúde, Ocupações e Grupos de Risco. (pp. 169194). Campinas: Papirus.

Rout, U. (1999). Gender Differences in Stress, Satisfaction and Mental Wellbeing among General Practitioners in England. Psychology, Health \& Medicine, 4(4), 345-354.

Rout, U. U. (2000). Stress amongst District Nurses: A Preliminary Investigation. Jounral of Clinical Nursing, 9, 303-309.

Rydstedt, L. W., Johansson, G., \& Evans, G. W. (1998). A Longitudinal Study of Workload, Health and Well-being among Male and Female Urban Bus Drivers. Journal of Occupational and Organizational Psychology, 71, 35-45.

Selye, H. (1982). History and Present Status of the Stress Concept. Em L. Goldberger \& S. Breznitz (Eds.), Handbook of Stress: Theoretical and Clinical Aspects. (pp. 7-17). New York: Free Pass. 
Sisto, F. F., Noronha, A. P. P., Santos, A. A. A., \& Baptista, M. N. (em construção). Escala de Sensibilidade ao Estresse.

Tyler, P. A., Carroll, D., \& Cunningham, S. E. (1991). Stress and Well-being in Nurses: A Comparison of the Public and Private Sectors. International Journal of Nursing Studies, 28(2), 125-130.

Zegans, L. S. (1982). Stress and the Development of Somatic Disorders. Em L. Goldberger \& S. Breznitz (Eds.), Handbook of Stress: Theoretical and Clinical Aspects. (pp. 134152). New York: Free Pass. 